- Link with voluntary services in local communities - e.g. Age UK, Macmillan Cancer Information.

Conclusion Consideration of role to support Primary Carer Networks and facilitate earlier discussions for people affected by a life limiting diagnosis.

\section{P-180 IDENTIFYING 'WHAT MATTERS?': AN ACT-BASED GROUP PROGRAMME FOR PEOPLE WITH CANCER}

${ }^{1}$ Angie Arnold, 'Jo Poyner, 'Rhiannan Lewis, ${ }^{2}$ Diane Playford, ${ }^{2}$ Carolyn Gordon, ${ }^{2}$ Georgina Slatter. ${ }^{1}$ The Shakespeare Hospice, Stratford upon Avon, UK; ${ }^{2}$ South Warwickshire Foundation Trust, Warwick, UK

10.1136/bmispcare-2019-HUKNC.202

Cancer and its treatment brings many challenges for patients and their families, not least physical and psychological recovery, and a return to work and/or activities of daily living (reintegration). Whilst developments in cancer treatment and a rise in survival rates has led to increased chances of people returning to work or other vocational/occupational activities, people with cancer continue to live with the long-term effects of the disease and/or its treatment. As a result, survivors may require some support to return to a more active life posttreatment, and evidence suggests that multi-disciplinary programmes, informed by CBT and Third Wave approaches are an appropriate intervention in this respect.

The poster will describe a collaborative project, undertaken by hospice and NHS services, which aimed to develop an ACT-based group programme. The poster will briefly outline the background to, and rationale for, the project before describing the development of a six-week programme, informed by Acceptance and Commitment Therapy (ACT). The group protocol was designed to support participants' identification of their core values, which some felt had been compromised because of cancer and treatment.

Subsequent content and activities enabled participants to take up, or return to, significant activities that were in line with, or a 'step towards', their values. The programme was delivered on three occasions to small groups and in total ten participants, referred from both hospice and NHS settings completed the full programme. Participant feedback and facilitators' reflections on the programme suggest it was a supportive process, leading to an increase in functioning and sense of self-efficacy and a small data set is available which supports this. The results of the group evaluation will be discussed, and recommendations and conclusions made.

\section{P-181 DEMENTIA TOGETHER - A LOCAL SERVICE EVALUATION}

${ }^{1}$ Ruth Strudwick, 'Katie Tyrell, 'Andreea Tocca, ${ }^{2}$ Jo Marshall, ${ }^{2}$ Alana Page, ${ }^{2}$ Sharon Barber. ${ }^{1}$ University of Suffolk, Ipswich, UK; ${ }^{2}$ Sue Ryder, Ipswich, UK

\subsection{6/bmjspcare-2019-HUKNC.203}

Background The Dementia Together Service was launched in April 2017 and by the end of December 2018 had registered and supported 2,202 people - both people living with dementia and their carers living across Suffolk. The service consists of a helpline and staff who visit people living with dementia and their carers, providing support and signposting them to other services.
Aim The service is led by Sue Ryder working with a number of local organisations in service delivery. Despite a comprehensive range of services across Suffolk, people living with dementia were overwhelmed by varying offers which disabled them from accessing the most appropriate services to meet their needs.

Methods A service evaluation was undertaken, based on the views of people living with dementia, their carers, Dementia Together staff and external stakeholders. Questionnaires and focus groups were utilised to obtain both quantitative and qualitative data. Purposive sampling was utilised for the focus groups with people living with dementia and carers, and all staff were included in their focus group. Data from all methods were analysed and triangulated.

Results During Phase 1 of the evaluation, 133 questionnaire responses were gathered from people living with dementia, their carers, Dementia Together staff and external stakeholders. Focus groups were carried out with 18 people living with dementia, carers and navigators.

In phase 2, further focus groups with 20 people living with dementia, carers and navigators were conducted along with three case study interviews $(n=3)$ and telephone/email interviews with eight stakeholders. Existing data from meetings and other reports were also analysed.

Conclusions The Dementia Together service has made a significant positive difference to people living with dementia, their families and carers across Suffolk.

\section{P-182 DEVELOPING A SUSTAINABLE MODEL OF CARE FOR PATIENTS WITH END-STAGE HEART FAILURE}

${ }^{1}$ Wendy Sturt, ${ }^{1}$ Gill Horne, ${ }^{2}$ Jo Sykes, ${ }^{2}$ Tim Chester. ${ }^{1}$ Rowcroft Hospice, Torquay, UK; ${ }^{2}$ Torbay and South Devon NHS Foundation Trust, Torbay, UK

\subsection{6/bmjspcare-2019-HUKNC.204}

Aims and objectives This 18 -month project aimed to enhance the opportunities for patients with end-stage heart failure (HF) and families to benefit from hospice and supportive care services to help them plan and experience better end-of-life care.

Method The HF service, care of older people, primary care, hospital palliative care, patients and families and hospice representatives, worked alongside a project lead designing a model of care and pathway for people with end-stage HF. A new Supportive and Palliative Care in Heart Failure MDT was established, providing an interdisciplinary forum for identification of those at the end-of-life, and aiding referral to specialist palliative care services. A patient information leaflet was designed and printed. Education was also provided for the HF Team on palliative/end-of-life care and end-stage HF management for the specialist palliative care services.

Outcomes/impact There is now an established pathway for patients, improved understanding and communications between the $\mathrm{HF}$ and specialist palliative care teams. The MDT meeting is held twice monthly and specialist palliative care referrals have more than doubled during the project. Patients and their families have access to core information about hospice and other end-of-life care services in their locality. Documentation audits of advance care planning discussion demonstrate that $64 \%$ of patients reviewed at the MDT had discussions about their wishes. However, more 